\title{
P04-3-5 Poster session
}

\section{Effects of deficiency of Kelch-like ECH-associated protein 1 on osteoclastogenesis by constitutive activation of nuclear factor E2 p45-related factor 2}

\author{
Eiko Sakai, Yu Yamaguchi, Yutaka Fukuma, Takayuki Tsukuba
}

Department of Dental Pharmacology, Nagasaki University Graduate School of Biomedical Sciences, Japan

[Background] Kelch-like ECH-associated protein 1 (Keap1) binds to nuclear factor E2 p45-related factor 2 (Nrf2), a transcription factor of antioxidant enzymes. Previous studies indicated that oxidative stresses stimulate osteoclastogenesis, which is responsible for bone loss, by activating receptor activator of NF-kappaB ligand (RANKL)-dependent pathways. In addition, recent studies reported that Nrf2 deficiency stimulates osteoclastogenesis. However, the effects of Keap1 deficiency remain unclear.

[Methods] Keap1-deficient-, Nrf2-deficient-, Keap1-Nrf2-double deficient mice were used for osteoclast formation.

[Results] By using Keap1-deficient newborn mice, we observed that osteoclast number was reduced and that talus and calcaneus bone formation was partially retarded without severe gross abnormalities in vivo. Moreover, Keap1-deficient macrophages were unable to differentiate into osteoclasts in vitro via attenuation of RANKL-regulated signaling and expression of nuclear factor of activated T cells-1 (NFATc1), a key transcription factor for osteoclastogenesis. During osteoclastogenesis, RANKL-induced gene expression of mitochondrial enzyme is required for down-regulation of IFN regulatory factor (IRF8), a negative transcriptional regulator of NFATc1. Our results indicate that Keap1 deficiency down-regulated peroxisome proliferator-activated receptor-gamma coactivator 1beta and mitochondrial gene expression and up-regulated Irf8 expression, as well as up-regulation of Mafb, a negative regulator of NFATc1. Furthermore, Keap1dependent phenotypes, such as attenuation of NFATc1 expression and the up-regulation of Irf8 as well as Mafb in Keap1deficient cells were reversed in Keap1-Nrf2-double deficient cells, suggesting that constitutive Nrf2 activation is responsible for the IRF8/MafB-mediated NFATc1 down-regulation and impaired osteoclastogenesis. These results also support the possibility that Nrf2 activators might have potential activity to inhibit osteoclast differentiation via NFATc1dependent pathways. To elucidate this possibility, we examined effects of tert-butylhydroquinone (tBHQ) and sulforaphane (SR), both are widely used as Nrf2 activators, on the differentiation of macrophages into osteoclasts. Both compounds inhibited the formation of osteoclasts in a dose-dependent manner, accompanied by increased levels of Irf8 and Mafb expression. Moreover, our observations with tBHQ and SR were reversed in Nrf2-deficient cells.

[Conclusion] These results suggest that the Keap1/Nrf2 axis plays important roles in NFATc1 expression and osteoclast differentiation. 